\title{
Diagnosing erectile dysfunction could save your patient's life
}

\author{
Gerald Brock, MD, FRCSC
}

Professor of Surgery, Urology Program Director, Western University; Chair, Office of Education, Canadian Urologic Association, London, ON

Cite as: Can Urol Assoc J 2014;8(7-8):S151-2. http://dx.doi.org/10.5489/cuaj.2311

Published online August 11, 2014.

\section{Abstract}

Erectile dysfunction and coronary artery disease are increasingly recognized as different clinical manifestations of the same process. Because the smaller vessels of the penis may be affected by plaque burden much earlier than the larger coronary, internal carotid, and femoral arteries, men may present with symptoms of erectile dysfunction long before signs of cardiovascular disease manifest. This presents an opportunity for the urologist to not only treat the sexual dysfunction and uncover occult coronary disease, but also to screen for cardiac risk, as even men with mild arteriogenic erectile dysfunction may be at risk for cardiovascular disease.

\section{Introduction}

Erectile dysfunction (ED) is increasingly being recognized as a vascular disorder, with predictive value for ensuing cardiac events equal to a strong family history or a history of smoking. Men presenting with ED often have risk factors for atherosclerosis and, conversely, ED is frequently reported in men with vascular syndromes, such as coronary artery disease (CAD), hypertension, cerebrovascular disease, peripheral arterial disease and diabetes mellitus. ${ }^{1}$ The association between ED and vascular disease lies with the vascular endothelium, which has an essential role in the regulation of circulatory function. Evidence suggests that endothelial dysfunction can be detected in men with ED well before overt manifestations of vascular damage, including atherosclerotic effects. ${ }^{2}$

Achieving and maintaining an erection require the proper functioning of both vascular and neurogenic components, which are in turn modulated by hormonal status and extrinsic factors. Disturbance of these systems lead to organic ED. Focal stenosis of the common penile artery can occur due to blunt pelvic or perineal trauma such as bicycling accidents. Veins may fail to close during the erection process, as a result of old age, diabetes mellitus or penile trauma (e.g., Peyronie's disease or penile fracture). Failure to initiate nerve impulse (or interrupted neural transmission) following a stroke, Alzheimer's disease, spinal cord injury, radical pelvic surgery or diabetic neuropathy may also cause ED.

Sensory involvement of the genitalia is essential to achieving and maintaining reflexogenic erection; this becomes increasingly important as the effect of psychogenic stimuli declines with age. Hormonal modulation can also lead to ED, as androgen deficiency decreases nocturnal erections and libido; to some degree, nitric oxide release in the corporal endothelium is androgen dependent. Hyperprolactinemia may also contribute to sexual dysfunction, as prolactin inhibits central dopaminergic activity, ultimately resulting in hypogonadism. Extrinsic factors for ED include antipsychotics, antidepressants and central-acting antihypertensive drugs, which all have the potential to perturb the central neurotransmitter pathways involved in sexual function. Beta-adrenergic blocking agents, thiazide diuretics and spironolactone can also contribute to ED. ${ }^{3}$ Smoking can also contribute to ED by inducing vasoconstriction and penile venous leakage due to its contractile effect on smooth muscle. As well, alcohol taken in large quantities or chronically over time can cause sedation and decreased libido. ${ }^{4-6}$

Essentially, ED and CAD are different clinical manifestations of the same spectrum of disease; however, because the penile arteries are smaller and require great flexibility, symptoms are likely to appear earlier than in the coronary or carotid arteries. The artery size hypothesis has been proposed to address this association. ${ }^{1}$ Given the systemic nature of atherosclerosis, one would expect that all major vascular beds would be affected to the same extent. However, symptoms usually appear at different points in the system at different times. This is likely because larger vessels are able to better tolerate the same amount of plaque than smaller ones. Coronary circulation is therefore not critically affected because of its larger size, which might explain why CAD is not evident in early-stage ED. Because of its smaller diameter, the penile artery will be severely narrowed if 50\% obstruction occurs, suggesting a mechanism for the frequent 


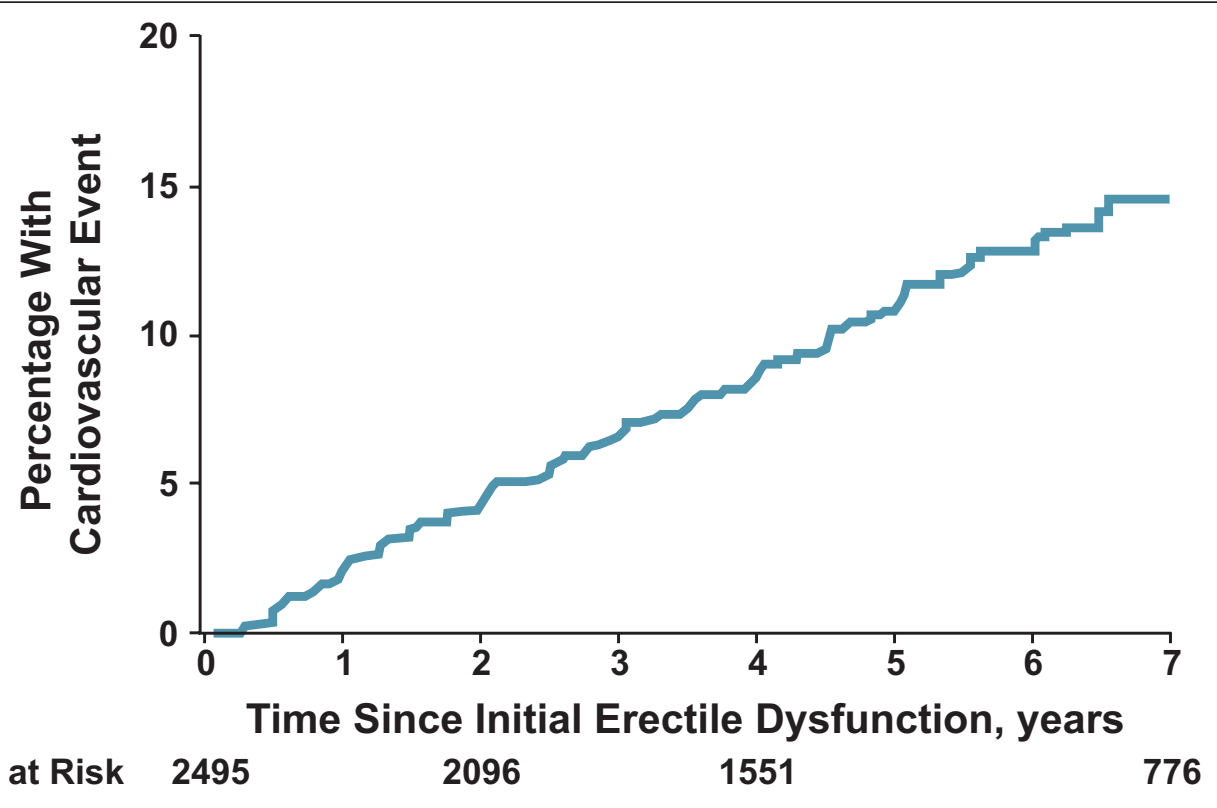

Fig. 1. Time to any cardiovascular event following an initial report of erectile dysfunction.? Copyright (ㄷ 2005. American Medical Association. All rights reserved.

coexistence of angina and ED. However, the plaque burden that produces significantly impaired circulation in the small arteries of the penis may cause only $30 \%$ to $40 \%$ occlusion of the larger coronary, internal carotid, and femoral arteries. Therefore, while atherosclerosis sufficient to trigger ED may not cause clinically apparent cardiovascular disease, progression of the atherosclerotic process in the larger arteries may subsequently result in $C A D$, stroke, or claudication. As a result, ED and CAD should be considered as two different aspects of the same disease.

The association between ED and the subsequent occurrence of $\mathrm{CV}$ disease was examined in men aged 55 years or older in the Prostate Cancer Prevention Trial (PCPT). ${ }^{7}$ Following an initial report of ED, $2 \%$ of men had experienced an initial CV event at 1 year, and $11 \%$ had experienced a CV event by 5 years (Fig. 1). The unadjusted risk of CV disease was 0.015 per person-year for men whose ED appeared after entry into the study, compared with 0.024 per person-year for those who had ED upon entry into the study, lending further support to the suggestion that ED may be an early manifestation of CV disease. The association between ED and CV disease was on a similar order to tobacco use or a family history of myocardial infarction.

Epidemiologic studies suggest that the predictive value of ED is stronger in younger men. In the Olmsted County Study of Urinary Symptoms and Health Status Among Men, men 40 to 49 years of age with ED had a 50-fold higher incidence of new-incident CAD than those without ED. However, ED was less predictive for CAD in men 70 years and older. $^{8}$

\section{Conclusion}

Sexual dysfunction may be the only reason men present to the doctor. This presents an opportunity, not only to treat the sexual dysfunction, but to also screen for cardiac risk. Even men with mild arteriogenic ED may be at risk for cardiovascular disease. It is important for these men to have their risk assessed early, before sequelae develop, and to manage any risks aggressively.

Competing interests: Dr. Brock has received honoraria from Eli Lilly, Coloplast, AMS, GSK, Abbott, Johnson and Johnson, Ferring and Actavis.

\section{References}

1. Montorsi P, Ravagnani PM, Galli S, et al. The artery size hypothesis: A macrovascular link between erectile dysfunction and coronary artery disease. Am J Cardiol 2005;96:19M-23M. http://dx.doi.org/10.1016/i. amicard.2005.07.006

2. Guay AT. Relation of endothelial cell function to erectile dysfunction: Implications for treatment. Am I Cardiol 2005;96(12B):52M-56M. http://dx.doi.org/10.1016/i.amicard.2005.10.006

3. Fazio L, Brock G. Erectile dysfunction: Management update. CMAJ 2004;170:1429-37. http://dx.doi. org/10.1503/cmaj.1020049

4. Lue TF. Erectile dysfunction. N Engl J Med 2000;342:1802-13. http://dx.doi.org/10.1056/ NEJM200006153422407

5. Miller TA. Diagnostic evaluation of erectile dysfunction. Am Fam Physician 2000;61:95-104, 109-10.

6. NIH Consensus Conference. Impotence. NIH Consensus Development Panel on Impotence. JAMA 1993;270:8390. http://dx.doi.org/10.1001/jama.1993.03510010089036

7. Thompson IM, Tangen $C M$, Goodman PJ, et al. Erectile dysfunction and subsequent cardiovascular disease. JAMA 2005;294:2996-3002. http://dx.doi.org/10.1001/jama.294.23.2996

8. Inman BA, Sauver JL, Jacobson DJ, et al. A population-based, longitudinal study of erectile dysfunction and future coronary artery disease. Mayo Clin Proc 2009;84:108-13. http://dx.doi.org/10.4065/84.2.108

Correspondence: Dr. Gerald Brock, St. Joseph's Health Centre, Department of Urology, 268 Grosvenor St., London, 0N N6A 4V2; gebrock@sympatico.ca 